\title{
Necesidades de capacitación de los productores agrarios en el altiplano de Puno, Perú
}

\author{
Training needs of agricultural producers in the highlands of Puno Peru
}

${ }^{1}$ Fermin Francisco Chaiña Chura

${ }^{2}$ Juan Inquilla Mamani
ORCID: 0000-0003-0559-9748

ORCID: 0000-0003-2540-9091

\section{RESUMEN}

El propósito de esta investigación fue analizar y comprender las necesidades de capacitación de los productores agrarios en el altiplano, centro poblado de Santa Bárbara de Moro del distrito de Paucarcolla Puno, Perú. La hipótesis de la investigación fue: "las variables socioeconómicas tienen relación con las necesidades de capacitación"; bajo un diseño de investigación correlacional y explicativo. Se utilizó el procedimiento metodológico cuantitativo, mediante la técnica de la encuesta y el instrumento del cuestionario, aplicado a 85 jefes de familia de una población de 108 familias, complementado con la observación directa. Los principales resultados fueron: los factores socioeconómicos tienen relación diferenciada con la demanda de capacitación; el $7.1 \%$ de jefes de familia requieren capacitación en temas de agricultura, conservación de suelos, medio ambiente y riego tecnificado; el 5.9\%, en producción agropecuaria; el 16.5\%, 5.9\%, 8.2\%, 11.8\%, 15.3\%, $9.4 \%$ en temas de ganadería, manejo, alimentación, sanidad, mejoramiento y derivados lácteos respectivamente; el $2.4 \%$ de jefes de familia en artesanía; el 3.5\% en temas de mercado y red vial; el 1.2\% en temas de salud y alimentación humana, similar proporción en liderazgo.

Palabras clave: Actores productivos, capacitación rural, familias, necesidades.

\section{ABSTRACT}

The objective of this research was to examine and understand the training needs of agricultural producers in the altiplano, the population center of Santa Bárbara de Moro in the district of Paucarcolla Puno, Peru. The investigation's hypothesis was: "socioeconomic variables are related to training needs"; under a correlational and explanatory research design. The methodological quantitative procedure was used, through the technique of the survey and the instrument of the questionnaire, applied to 85 heads of family of a population of 108 families, complemented with direct observation. The most important results were: socioeconomic factors have a differentiated relation with training demand; $7.1 \%$ of heads of family require training in agriculture, soil conservation, environment and technified irrigation; $5.9 \%$, in agricultural production; $16 \% .5 \%, 5.9 \%, 8.2 \%$, $11.8 \%, 15.3 \%, 9.4 \%$ in the areas of livestock, management, nutrition, health, improvement and dairy products, respectively; $2.4 \%$ of heads of households are involved in handicrafts; $3.5 \%$ are involved in the market and road network; $1.2 \%$ are involved in health and human nutrition, and a similar proportion are leaders.

Keywords: Families, needs, productive actors, rural training.

${ }^{1}$ Universidad Nacional del Altiplano. Facultad de Ciencias Sociales. Puno, Perú- E-mail: fchaina@unap.edu.pe

${ }^{2}$ Universidad Nacional del Altiplano. Facultad de Ciencias Sociales. Puno, Perú- E-mail: jinquilla@unap.edu.pe 


\section{INTRODUCCIÓN}

En términos de desarrollo sostenible en contextos agrarios, el propósito no solo es la búsqueda de la productividad, sino la mejora en la calidad de vida de la población rural y en la sustentabilidad de los sistemas de producción (Rendón, Roldán, Hernández y Cadena, 2014). En efecto, la investigación de las necesidades de capacitación de los actores productivos del medio rural (Curró, Pozzolo, Fuica y Bogliani, 2012) es importante debido a que es necesario conocer las urgencias de conocimientos y tecnologías para fortalecer el funcionamiento de las economías familiares; para lo cual es necesario contar con información precisa sobre estas necesidades (Maya, Ángeles y Camarena, 2014).

Los requerimientos de capacitación identificados, analizados y priorizados servirán de base para diseñar procesos de educación no formal a fin de impulsar la atención de las necesidades de la población rural materia de desarrollo de capacidades (Sinacatri, 2007); diseño de líneas de acción eficaces y eficientes en la estrategia de capacitación (Posada y Vaca, 2001); en la búsqueda de equidad e igualdad social en cuestiones de ingresos y bienestar social (Juárez, 2012).

Iturbide (2012) considera que el estudio de necesidades de capacitación es una herramienta de trabajo por medio del cual se recopila información sobre el estado actual de "conocimiento y gestión" de un contexto determinado, bajo los parámetros social, económico y político; concordante con Posada y Vaca (2001), al considerar que el objetivo central es captar las necesidades implícitas y explícitas de capacitación para la mejora de las condiciones de trabajo y de vida en este sector de actividad.

En esa perspectiva, el fortalecimiento de las capacidades humanas es de vital importancia para el desarrollo del agro. Por tanto, la capacitación del capital humano es importante para tener una visión adecuada y los conocimientos necesarios para asegurar la sostenibilidad de los sistemas de producción agropecuaria (Mesa y Machado, 2009). En efecto, impulsar la construcción de bienestar social que se fundamenta, entre otros aspectos, en la capacidad para introducir innovaciones sociales, institucionales y culturales, así como en la base productiva y el tejido empresarial de un territorio (Rendón et al. 2015). No es suficiente enseñar impartiendo contenidos formales, sino desarrollar aptitudes y destrezas a través de experiencias prácticas, aprender haciendo, a fin de que la práctica resulte productiva (Fernández y Peña, 2012).

En ese contexto, en el altiplano de Puno, las pampas de Moro es un espacio geo-social muy especial. Al constituirse sobre la base de hacienda, como beneficiarios de la Ley de Reforma Agraria $\mathrm{N}^{\circ}$ 17716, organizada en empresa asociativa, luego en comunidad y hoy como centro poblado. Cuya estructuración en economías familiares se desarrolla con velocidad por sus condiciones geográficas favorables para actividades agropecuarias, principalmente ganadería vacuna por la cobertura de pastos naturales, complementado con la instalación de pastos cultivados y la accesibilidad vial a dos de los centros urbanos más dinámicos de la región: Puno y Juliaca.

De modo que, dicho asentamiento humano se constituye en una nueva composición social y cultural heterogénea con sus diferentes comportamientos. Como consecuencia de los cambios en la estructura agraria del país, la política de reestructuración y desintegración de la Sociedad Agrícola de Interés Social Buena Vista (SAIS), donde los socios titulares de la organización comunal proceden de los distritos de Paucarcolla, Huata, Atuncolla y Caracoto, para constituir la organización comunal, logrando superar las diferencias y propiciar la integración por la necesidad de sostenibilidad en sus propósitos de desarrollo de capacidades, personal, familiar y local. 
Este proceso adquiere una nueva perspectiva de desarrollo que direcciona hacia el mejoramiento de las condiciones de vida de la mayoría de la población (Iglesias, 2014; Lutz, 2017; Hernández, Herrera y Chávez, 2015), en concordancia con la Agenda Bicentenario del Perú 2021 que considera como una oportunidad para pensar y hacer el país que queremos ser, y forjar una nueva ciudadanía preparada para afrontar los desafíos de este siglo, fortaleciendo nuestra identidad de "ser peruano" en las oportunidades que nos depara el futuro (Gobierno peruano, 2018). Por esa razón, se trata de procesos que indican transformación en la organización del territorio para el desarrollo con base agropecuario. En este marco, se aspira a una comunidad rural con capacidades personales, locales y eficientes en la gestión de sus unidades de producción familiar; de igual modo, concordante con la Agenda 2030 definida a partir de la Organización de las Naciones Unidas para la Educación (UNESCO). De hecho, busca en primer lugar y ante todo canalizar el mundo hacia un camino sostenible (Alencastro, Castañón, Quiñores y Egas, 2020), en la búsqueda del bienestar de la población (Mora, 2013); a pesar de su relevancia, uno de los aspectos aún débiles es la capacitación (Quispe, 2006).

Es así que, el problema está en que las acciones de capacitación en contextos rurales se originan desde el sector institucional y no a partir de la expresión de los verdaderos interesados de los actores productivos locales; el reconocimiento de las prioridades e intereses de los beneficiarios deben reorientarse hacia un trabajo más horizontal y dialógico (Landini, Bianqui y Russo, 2014).

Con base a lo expuesto, el objetivo de esta investigación fue analizar las necesidades de capacitación en función a los factores sociales y económicos, componentes de la estructura y funcionamiento de estas economías familiares rurales, siendo necesario resaltar las propuestas en el diseño de eventos de capacitación que contribuya a la población hacia el logro del desarrollo sostenible en dicho territorio.

\section{MATERIAL Y MÉTODOS}

El estudio se realizó en el altiplano de Puno, espacio altoandino donde se encuentran ubicadas las pampas de Moro en el centro poblado de Santa Bárbara, en la zona Noreste del distrito de Paucarcolla, provincia y región Puno; dedicada principalmente a la agricultura y crianza de ganado, a una altitud de $3812 \mathrm{~m}$ de altitud.

El tipo de investigación es correlacional y explicativo (Hernández, Fernández y Baptista, 2014), por la naturaleza del estudio que analiza las necesidades de capacitación en un contexto rural. De una población de 108 familias (INEI, 2017), se determinó intencionalmente una muestra no probabilística, seleccionando arbitrariamente a 85 jefes de familia, que brinden información que permita comprender los factores sociales y económicos que condicionan las necesidades de adiestramiento de los actores productivos.

La investigación se desarrolla utilizando métodos cuantitativos, para analizar la relación existente entre las variables socioeconómicas y los requerimientos de capacitación. Los ejes de análisis son las características socioeconómicas de las unidades familiares y necesidades de capacitación; las dimensiones de análisis constituidas por la interacción social y la dimensión económica. La unidad de análisis son las necesidades de capacitación y la unidad de observación está constituida por el jefe de familia.

La técnica y el instrumento de recolección de información fueron: la encuesta y el cuestionario, complementado con la observación directa. La investigación se desarrolló en tres momentos; primero, se realizó la revisión documental a cerca de demandas de capacitación; segundo, se hizo la colecta de información en el campo a partir de los jefes de familia, mediante la observación; y finalmente, el procesamiento de la información en una base de datos, para la construcción de estadísticas y 
las respectivas tablas, lo que permitió organizar la información para el análisis y la discusión.

\section{RESULTADOS}

\section{Características sociales y económicas de los actores productivos}

Las variables sociales estuvieron constituidas por la edad, sexo, estado civil, tamaño de la familia y nivel educativo. El tamaño del predio, tenencia de ganado y el ingreso constituyen las variables económicas. La variable de grupos etarios comprende entre 18 y 85 años de edad; donde predominan los jefes de familia entre las edades de 40 y 49 años, seguida de 30 a 39, de 50 a 59 y más de 70 años de edad; en correspondencia porcentual de $27.5 \%, 23.5 \%$, $21.2 \%$ y $10.6 \%$ respectivamente; en menor proporción entre las edades comprendidas de 60 a 69, 18 a 29 años. La variable sexo es importante para conocer las necesidades de capacitación. En esta investigación participaron 55 varones y 30 mujeres, que representan el $64.7 \%$ y $35.3 \%$ respectivamente.

La composición familiar está referida al tamaño de los miembros que la integran, siendo base fundamental para la organización de la producción. Las unidades familiares están constituidas por padres e hijos, y en algunos casos los miembros de la familia viven en las ciudades de Puno y Juliaca principalmente, por la cercanía y accesibilidad. No obstante, en algunos casos, en los caseríos queda solo un miembro al cuidado de la vivienda y del ganado familiar.

\section{Tabla 1}

Composición familiar y nivel educativo del jefe de familia

\begin{tabular}{cccccc}
\hline \multicolumn{4}{c}{ Composición familiar } & \multicolumn{3}{c}{ Estudios alcanzados } \\
\hline Integrantes & Frecuencia & Porcentaje & Nivel & Frecuencia & Porcentaje \\
\hline$<=1$ & 5 & 5.9 & Sin estudios & 4 & 4.7 \\
$2-3$ & 28 & 32.9 & Primaria & 37 & 43.5 \\
$4-5$ & 39 & 45.9 & Secundaria & 34 & 40.0 \\
$6-7$ & 9 & 10.6 & Superior & 9 & 10.6 \\
$8+$ & 4 & 4.7 & Téc. superior & 1 & 1.2 \\
Total & 85 & 100.0 & Total & 85 & 100.0 \\
\hline
\end{tabular}

Fuente: Información obtenida del cuestionario.

En la Tabla 1 se presenta la relevancia de unidades familiares en los niveles 4 a 5 integrantes $(45.9 \%)$ y 2 a 3 integrantes (32.9\%); seguida por el nivel de 6 a 7 miembros que representa el $10.6 \%$ de familias y el $5.9 \%$ de productores viven solos. Aunado a esto, el nivel educativo se refiere al número de años de estudio en educación formal alcanzado por el jefe de familia. Se demuestra la predominancia de niveles educativos de primaria y secundaria, seguido de educación superior, el $4.7 \%$ no participó en educación formal o ha dejado de estudiar en los primeros grados.
En este contexto, los predios de los productores presentan diferentes dimensiones. Ubicados en espacios geográficos distantes, en algunos casos en otro distrito, pero dentro de la provincia de Puno; la tierra resulta ser un medio de producción fundamental para la actividad agropecuaria. La Tabla 2 refleja la predominancia de predios menores de 5 hectáreas en una proporción de 89.4 \%; seguido por el nivel de 11 a 15 y de 6 a 10 hectáreas en proporción de $5.9 \%$ y $2.4 \%$ respectivamente; y el $2.4 \%$ de productores cuentan con más de 26 hectáreas. 
Tabla 2

Superficie del predio familiar

\begin{tabular}{ccccc}
\hline $\begin{array}{c}\text { Tamaño del predio } \\
\text { (hectáreas) }\end{array}$ & Frecuencia & Porcentaje & $\begin{array}{c}\text { Porcentaje } \\
\text { válido }\end{array}$ & $\begin{array}{c}\text { Porcentaje } \\
\text { acumulado }\end{array}$ \\
\hline$<=5$ & 76 & 89.4 & 89.4 & 89.4 \\
$6-10$ & 2 & 2.4 & 2.4 & 91.8 \\
$11-15$ & 5 & 5.9 & 5.9 & 97.6 \\
$26+$ & 2 & 2.4 & 2.4 & 100.0 \\
Total & 85 & 100.0 & 100.0 & \\
\hline
\end{tabular}

Fuente: Información obtenida del cuestionario.

En virtud de los resultados, la presencia de productores rurales con predios menores de 5 hectáreas permiten caracterizar la persistencia de economías familiares que conceptualmente son consideradas como economía campesina; constituida por un conjunto de elementos que se relacionan entre sí como la agricultura, la ganadería, los procesamientos para el consumo de la familia y para el mercado, en perspectiva de generación de ingresos económicos por venta de productos.

El ingreso mensual de los productores se presenta en los siguientes niveles: en el extremo inferior los que tienen un ingreso mensual menores de $\mathrm{S} / 20$ y en el nivel superior los que tienen más de $\mathrm{S} / 1221$.

\section{Tabla 3}

Ingreso mensual de la unidad familiar

\begin{tabular}{ccccc}
\hline Nivel de ingreso & Frecuencia & Porcentaje & $\begin{array}{c}\text { Porcentaje } \\
\text { válido }\end{array}$ & $\begin{array}{c}\text { Porcentaje } \\
\text { acumulado }\end{array}$ \\
\hline$<=20$ & 6 & 7.1 & 7.1 & 7.1 \\
$21-320$ & 32 & 37.6 & 37.6 & 44.7 \\
$321-620$ & 19 & 22.4 & 22.4 & 67.1 \\
$621-920$ & 11 & 12.9 & 12.9 & 80.0 \\
$921-1220$ & 7 & 8.2 & 8.2 & 88.2 \\
$1221+$ & 10 & 11.8 & 11.8 & 100.0 \\
Total & 85 & 100.0 & 100.0 & \\
\hline
\end{tabular}

Fuente: Información obtenida del cuestionario.

Según la tabla precedente, existe la predominancia de ingresos mensuales familiares de S/ 21 a S/ 320 y S/ 321 a S/ 620 en la proporción de $37.6 \%$ y $22.4 \%$ respectivamente; seguido por otro grupo de ingresos que varía entre S/ 621 a S/ 920 y S/ 921 a $\mathrm{S} / 1220$ respectivamente. Es notorio un grupo de productores con ingresos mensuales superiores a S/ 1221 en una proporción de 11. $8 \%$; el $7.1 \%$ de productores no tiene ingresos mensuales fijos, ellos en algunos casos solo tienen ingresos al año, por la venta de ganado principalmente, cuya saca es anual o cada dos años.

Las variables sociales están constituidas por las características personales, familiares y sociales propiamente dichas; las condiciones económicas que tienen como componentes el tamaño del predio, tenencia de ganado y el ingreso mensual; cuyos promedios se presentan en la Tabla 4. 
Necesidades de capacitación de los productores agrarios en el altiplano de Puno, Perú

Julio-diciembre 2020

Tabla 4

Características de las variables socioeconómicas

\begin{tabular}{lrrrrrrr}
\hline $\begin{array}{l}\text { Medidas } \\
\text { estadísticas }\end{array}$ & Sexo* & Edad & $\begin{array}{l}\text { Tamaño } \\
\text { de la } \\
\text { familia }\end{array}$ & $\begin{array}{l}\text { Nivel } \\
\text { educativo } \\
\text { (años) }\end{array}$ & $\begin{array}{l}\text { Tamaño } \\
\text { Predio } \\
\text { (ha) }\end{array}$ & $\begin{array}{l}\text { Tenencia } \\
\text { ganado } \\
\text { vacuno** }\end{array}$ & $\begin{array}{l}\text { Ingreso } \\
\text { mes } \\
(\mathrm{S} /)\end{array}$ \\
\hline Media & 00.00 & 47.81 & 4.09 & 8.85 & 2.95 & 11.91 & 588.48 \\
Mediana & 1.00 & 47.00 & 4.00 & 11.00 & 1.00 & 10.00 & 400.00 \\
Moda & 1 & 35 & 4 & 6 & 1 & 8 & 200 \\
Des estándar & .82 & 14.93 & 1.93 & 3.823 & 4.96 & 9.53 & 602.03 \\
Mínimo & 1 & 18 & 1 & 1 & 0 & $3 * *$ & 55 \\
Máximo & 2 & 85 & 12 & 16 & 30 & 55 & 3000 \\
\hline
\end{tabular}

*: La variable sexo: 55 hombres y 30 mujeres; **: existen pobladores que no crían ganado Fuente: Información obtenida del cuestionario.

La media de la edad del jefe de familia es 48 años, su unidad familiar está compuesto por 4 integrantes, el nivel educativo alcanzado es 9 años de escolaridad, la media del tamaño del predio es 3 hectáreas, son criadores de ganado vacuno cuyo promedio es 12 unidades y el ingreso por mes es $\mathrm{S} / 588.48$.

\section{Necesidades de capacitación de los actores productivos}

Se hace necesario resaltar la necesidad de capacitación a la población rural en el proceso de sus actividades productivas y su demanda que constituye la resultante de la expresión de los propios productores, en el marco de su vocación de criadores de ganado por las particularidades socioeconómicas y geográficas de su territorio, que presenta suelos cubiertos de pastos naturales aptos para la crianza de ganado vacuno y producción de leche; consiguientemente, su comercialización les genera ingreso económico familiar seguro, que en algunos casos es diario. Las necesidades de capacitación está en función a la actividad productiva desarrollada.

\section{Tabla 5}

Necesidades de capacitación en Moro

\begin{tabular}{lcccc}
\hline Necesidades de capacitación & Frecuencia & Porcentaje & $\begin{array}{c}\text { Porcentaje } \\
\text { válido }\end{array}$ & $\begin{array}{c}\text { Porcentaje } \\
\text { acumulado }\end{array}$ \\
\hline Agricultura y conservación de suelos & 4 & 4.7 & 4.7 & 4.7 \\
Medio ambiente y riego tecnificado & 2 & 2.4 & 2.4 & 7.1 \\
Producción agropecuaria & 5 & 5.9 & 5.9 & 12.9 \\
Ganadería & 14 & 16.5 & 16.5 & 29.4 \\
Manejo de ganado & 5 & 5.9 & 5.9 & 35.3 \\
Alimentación, pastos y forrajes & 7 & 8.2 & 8.2 & 43.5 \\
Sanidad animal & 10 & 11.8 & 11.8 & 55.3 \\
Mejoramiento de ganado e inseminación & 13 & 15.3 & 15.3 & 70.6 \\
Lácteos y procesamiento & 8 & 9.4 & 9.4 & 80.0 \\
Artesanía & 2 & 2.4 & 2.4 & 82.4 \\
Red vial y mercado & 3 & 3.5 & 3.5 & 85.9 \\
Salud y alimentación humana & 1 & 1.2 & 1.2 & 87.1 \\
Liderazgo & 1 & 1.2 & 1.2 & 88.2 \\
Otra necesidad & 10 & 11.8 & 11.8 & 100.0 \\
Total & 85 & 100.0 & 100.0 & \\
\hline
\end{tabular}

Fuente: Información obtenida del cuestionario. 
En la Tabla 5 se observan los requerimientos de capacitación de los actores productivos de Moro, donde el $7.1 \%$ de jefes de familia requiere capacitación en temas de agricultura, conservación de suelos, medio ambiente y riego tecnificado; el $5.9 \%$ en producción agropecuaria; el 16.5\%, $5.9 \%, 8.2 \%, 11.8 \%$, $15.3 \%$ y $9.4 \%$ en crianza de ganado en el manejo adecuado, alimentación, sanidad, mejoramiento y derivados lácteos respectivamente; para la actividad artesanal, el $2.4 \%$ de jefes de familia, el $5.5 \%$ en temas de mercado y red vial, el $1.2 \%$ en temas de salud y alimentación humana, similar proporción en liderazgo; y finalmente el $11.8 \%$ tienen otros requerimientos o no están interesados en recibir capacitación. Los resultados evidencian la predominancia de necesidades de capacitación en temas de ganadería, la naturaleza de la demanda expresa la necesidad de fortalecimiento de la actividad en la crianza de ganado vacuno, debido a las condiciones socio productivo y eco geográficas del medio.

\section{Relación entre variables socioeconómicas y necesidades de capacitación}

La verificación de la hipótesis se desarrolla en base a la información obtenida vía cuestionario; se postula que las variables sociales y económicas tienen relación con las necesidades de capacitación en el centro poblado de Santa Bárbara de Moro.

La hipótesis estadística:

Ho: Las variables sociales son independientes de las necesidades de capacitación.

Ha: Las variables sociales no son independientes de las necesidades de capacitación.

El análisis se realiza con la verificación de las hipótesis estadísticas, a través de la prueba de chi-cuadrada de Pearson, lo que permite determinar si existe asociación entre las variables, los resultados son:

Tabla 6

Variable estado civil y necesidades de capacitación en salud y alimentación humana

\begin{tabular}{lrrc}
\hline & & \multicolumn{2}{c}{$\begin{array}{c}\text { Significación asintótica } \\
\text { (2 caras) }\end{array}$} \\
\hline Chi-cuadrado de Pearson & Valor & gl & .006 \\
Razón de verosimilitud & $33.960^{\mathrm{a}}$ & 16 & .280 \\
N de casos válidos & 18.789 & 16 & \\
\hline
\end{tabular}

Nota: Tabla elaborada según la información del cuestionario y prueba Chi-cuadrado.

En la Tabla 6 se observa que el valor de la significación asintótica es menor a 0.05 , lo que indica que existe asociación entre la variable estado civil y necesidades de capacitación en salud y alimentación humana; por lo que se acepta la hipótesis alterna; la situación civil de los jefes de familia es importante en la determinación de los requerimientos de capacitación en la prevención de la salud y alimentación humana, artesanía, mercado, red vial, entre otros. 


\section{Tabla 7}

Variables sociales y necesidades de capacitación

\begin{tabular}{lccc}
\hline Chi - cuadrado de Pearson y variables & Valor & gl & $\begin{array}{c}\text { Significación } \\
\text { Asintótica } \\
\text { (2 caras) }\end{array}$ \\
\hline Chi - cuadrado de Pearson: & & & \\
1. Edad y n* en agricultura & $107.518^{\mathrm{a}}$ & 117 & .724 \\
2. Edad y nc en ganadería & $231.487^{\mathrm{a}}$ & 234 & .534 \\
3. Edad y nc en prev**, otros & $111.465^{\mathrm{a}}$ & 156 & .997 \\
4. Sexo y nc en agricultura & $1.081^{\mathrm{a}}$ & 3 & .782 \\
5. Sexo y nc en ganadería & $13.886^{\mathrm{a}}$ & 6 & $\mathbf{. 0 3 1}$ \\
6. Sexo y nc en prev, otros & $3.992^{\mathrm{a}}$ & 4 & .407 \\
7. E civil y nc en agricultura & $17.813^{\mathrm{a}}$ & 12 & .121 \\
8. E civil y nc en ganadería & $28.930^{\mathrm{a}}$ & 24 & .223 \\
9. E civil y nc en prev, otros & $33.960^{\mathrm{a}}$ & 16 & $\mathbf{. 0 0 6}$ \\
10. Com fam*** y nc en agricultura & $27.371^{\mathrm{a}}$ & 30 & .604 \\
11. Com fam y nc en ganadería & $41.820^{\mathrm{a}}$ & 60 & .964 \\
12. Com fam y nc en prev, otros & 105.614 & 40 & $\mathbf{. 0 0 0}$ \\
13. Nivel edu y nc en agricultura & $4.053^{\mathrm{a}}$ & 12 & .982 \\
14. Nivel edu y nc en ganadería & $29.916^{\mathrm{a}}$ & 24 & .188 \\
15. Nivel edu y nc en prev, otros & $13.711^{\mathrm{a}}$ & 16 & .620 \\
\hline
\end{tabular}

*: Necesidades de capacitación, **: Prevención de salud humana, otros, ***: Composición familiar. Nota: Tabla elaborada según la información del cuestionario y prueba Chi-cuadrado.

En la Tabla 7 se observan los resultados de la relación de las variables sociales y necesidades de capacitación en tres componentes de la unidad de producción familiar, la agricultura, la ganadería y la prevención de la salud y alimentación, artesanía, mercado, entre otros. De las quince pruebas, solo tres son representativas con valor de significación asintótica menor a 0.05 ; en el marco de este parámetro, la variable sexo tiene relación con necesidades de capacitación en ganadería referido al proceso de crianza de ganado vacuno. Por otro lado, las variables estado civil y composición familiar tienen relación con prevención de la salud y alimentación humana, artesanía, mercado, red vial, entre otros; en los otros casos no hay relación significativa.

Lahipótesis estadística:

Ho: Las variables económicas son independientes de las necesidades de capacitación.
Ha: Las variables económicas no son independientes de las necesidades de capacitación.

En la Tabla 8 de manera similar, al aplicar la prueba Chi-cuadrada de Pearson para verificar el nivel de asociación entre las variables económicas y las necesidades de capacitación, se constata que de las nueve pruebas solo dos son representativas con valor de asociación significativa, dado que el valor es menor a 0.05.

Al cumplir este parámetro, la tenencia de tierra tiene relación con necesidades de capacitación en la agricultura; además, el ingreso con necesidades de capacitación en temas de prevención de la salud y alimentación humana, artesanía, mercado, red vial, entre otros. 


\section{Tabla 8}

Variables económicas y necesidades de capacitación

\begin{tabular}{lrrr}
\hline Chi - cuadrado de Pearson y variables & Valor & gl & $\begin{array}{c}\text { Significación } \\
\text { Asintótica } \\
(2 \text { caras })\end{array}$ \\
\hline Chi - cuadrado de Pearson: & & & \\
1. Tenencia de tierra y nc* en agricultura & $49.271^{\mathrm{a}}$ & 33 &. $\mathbf{0 3 4}$ \\
2. Tenencia de tierra y nc en ganadería & $75.888^{\mathrm{a}}$ & 66 & .190 \\
3. Tenencia de tierra y nc en prev**, otros & $13.393^{\mathrm{a}}$ & 44 & 1.000 \\
4. Tenencia de ganado y nc en agricultura & $64.307^{\mathrm{a}}$ & 81 & .913 \\
5. Tenencia de ganado y nc en ganadería & $185.083^{\mathrm{a}}$ & 162 & .103 \\
6. Tenencia de ganado y nc en prev, otros & $76.382^{\mathrm{a}}$ & 108 & .991 \\
7. Ingreso y nc agricultura & $59.607^{\mathrm{a}}$ & 72 & .851 \\
8. Ingreso y nc ganadería & $168.062^{\mathrm{a}}$ & 144 & .083 \\
9. Ingreso y nc prev, otros & 124.257 & 96 & $\mathbf{0 2 8}$ \\
\hline
\end{tabular}

nc* : Necesidades de capacitación, prev**: Prevención de la salud humana, otros.

Nota: Tabla elaborada según la información del cuestionario y prueba Chi-cuadrado.

Por consiguiente, las variables sociales y económicas tienen relación diferenciada con las necesidades de capacitación en temas de agricultura, ganadería y prevención de la salud y alimentación humana, artesanía, mercado, red vial, entre otros.

\section{DISCUSIÓN}

\section{Variables socioeconómicas y necesidades de capacitación}

La relación de las variables sociales y las necesidades de capacitación es diversificada; al evidenciar que la variable sexo tiene relación con la demanda de conocimiento y tecnologías en ganadería; mientras que, el estado civil y la composición familiar tiene relación con las necesidades de capacitación en prevención de la salud y alimentación humana. En lo referente a las variables económicas, se encontró que la tenencia de tierra e ingreso tienen relación con las necesidades de capacitación en agricultura y en prevención de la salud y alimentación humana respectivamente.

Los resultados demuestran necesidades de capacitación en temas de ganadería, manejo de ganado, alimentación, pastos y forrajes, así como la sanidad animal, mejoramiento de ganado e inseminación artificial y procesamiento de derivados lácteos en temas de agricultura, conservación de suelos, medio ambiente además, en temas sociales referidos a la salud y alimentación humana, entre otros.

Las necesidades identificadas revelan el requerimiento de nuevos conocimientos, habilidades y actitudes; causa que genera los problemas que enfrentan los productores, cuya solución podría lograr impactos importantes en estas unidades de producción (Mesa y Machado, 2009). Por consiguiente, la adopción de tecnologías sostenibles exige capacitación de los actores locales a través de un programa ajustado a sus necesidades productivas.

En líneas generales, la perspectiva es diseñar procesos de capacitación coordinada con actores institucionales, quienes gestionan las políticas públicas, $\mathrm{y}$ actores productivos que son los interesados en su propio desarrollo, con enfoques de desarrollo que sean pertinentes y aplicables (Bauman, Bruno, Cleary, Dubois y Flores, 2004) en base a conocimientos locales (García, 2012), a través de prácticas adecuadas a las necesidades y particularidades de la población (Brumat, 2015), con el propósito centrado en la búsqueda de la productividad, en la mejora en la calidad de vida de la población rural y en la sustentabilidad de los sistemas de producción (Rendón et al, 2014; Monsalvo et al, 2017). 
En la capacitación, como proceso relacionado con el contexto local, es importante contar con más del $50 \%$ de prácticas en campo, incluir intercambios de experiencias con técnicos y productores locales y contar con profesionales expertos y productores reconocidos (FAO, 2018); adicionalmente, Gavito et al. (2017) proponen fomentar el trabajo de investigación en laboratorios sin muros, en los cuales grupos flexibles de actores diseñen, prueben y evalúen innovaciones tecnológicas que respondan a problemáticas ambientales y socio ecológicas locales y regionales específicas.

\section{Capacitación rural como educación no formal}

Las demandas de capacitación identificadas constituyen al hombre, pequeño productor, como el actor principal, siendo ellos los verdaderos interesados y conocedores de sus necesidades, de sus urgencias de conocimiento de tecnologías que requiere la estructura y funcionamiento de su unidad de producción familiar y como tal expresan la ayuda que requieren del sector institucional.

Entonces, la expresión de necesidades a partir de los productores rurales contribuirá al éxito de las acciones de capacitación; de lo contrario, puede resultar ajena a sus intereses y conducir al fracaso de la intervención vía capacitación. Por lo que, las acciones de capacitación no tendrían resultados adecuados, sus efectos e impactos generarían mayores problemas; nuestra intervención en lugar de ayudar resultaría siendo un factor que entorpece el desarrollo de estas economías, debido a que los contenidos de los eventos de educación no formal no solucionarían los problemas en sus unidades de producción familiar.

Para Raczynski y Román (2014), el ser rural en cualquier parte de América Latina conlleva menores oportunidades educativas para acceder al conocimiento en igualdad de condiciones a una real inserción en la sociedad global, para beneficio propio, de sus comunidades y del país; la pertinencia de la educación para los adultos tiene que ver con contenidos de enseñanza que aproximen la educación rural a las actuales realidades de estas zonas, para asegurar más y mejores oportunidades a estos grupos (Gajardo, 2014). Rodríguez, Sánchez y Armenta (2007) consideran que estas zonas rurales son un sector subdesarrollado dentro de los países en desarrollo; el problema es el tipo de futuro que le espera a este mundo rural (García, 2000).

En efecto, las poblaciones del medio rural plantean grandes desafíos para lograr el desarrollo de un futuro sostenible (Monsalvo et al, 2017); tales como las demandas de educación no formal en perspectiva del fortalecimiento del desempeño del capital humano (Bruno, Centurión, Robleda y Pérez, 2015); concordante con Juárez (2012), en la búsqueda de una mayor equidad e igualdad social en cuestiones de ingresos y bienestar social; para vivir en armonía con la naturaleza (Martínez y Carballo, 2013).

Asimismo, en estos contextos es necesario trabajar bajo la metodología "aprender haciendo y enseñar demostrando", que se materializa en la "práctica productiva", y que consiste en desarrollar actividades de campo (Fernández y Peña, 2012) en la búsqueda de procesos de aprendizaje significativos (Peirano, Estévez y Astorga, 2015), para promover el mejoramiento de las condiciones socioeconómicas de los habitantes de estas tierras (Pastor, Abraham y Torres, 2005).

En virtud de los resultados, las acciones de capacitación deben desarrollarse dentro de una concepción horizontal de diálogo entre los productores y los integrantes del sector institucional, para emprender eventos de fortalecimiento de capacidades para contribuir al mejoramiento de estos sistemas de producción como elemento de sensibilidad para el desarrollo de capacidades personales, familiares y locales. 


\section{CONCLUSIONES}

Las variables socioeconómicas tienen relación diferenciada con las necesidades de capacitación de la población rural en el centro poblado de Santa Bárbara de Moro en el altiplano de Puno; es evidente la predominancia de necesidades de capacitación en temas de ganadería. La naturaleza de la demanda expresa la necesidad de fortalecimiento de la actividad en la crianza de ganado, debido a las condiciones socio productivas y eco-geográficas del medio, específicamente en temas de manejo de ganado, alimentación apropiada, innovación tecnológica en el manejo de pastos y forrajes, sanidad animal, mejoramiento e inseminación artificialy procesamiento de derivados lácteos.

La necesidad de capacitación se expresa en menor proporción en temas de agricultura, conservación de suelos, riego tecnificado, temas ambientales; en temas sociales referidos a la salud y alimentación humana, así como liderazgo entre otros.

\section{REFERENCIAS BIBLIOGRÁFICAS}

Alencastro, A., Castañón, J., Quiñores, M. y Egas, F. (2020). Planificación estratégica para el desarrollo territorial de la Provincia Esmeraldas en Ecuador. Revista de Ciencias Sociales (Ve), XXVI (3) ,130-147. http://dx.doi.org/10.31876/rcs.v26i3

Bauman, P., Bruno, M., Cleary, D., Dubois, O. y Flores, X. (2004). Aplicación de estrategias de desarrollo enfocadas hacia las personas en el ámbito de la $F A O$. Núm.15. Recuperado de: http://www.fao.org/3/a-j3137s.pdf

Brumat, M. (2011). Maestros rurales: condiciones de trabajo, formación docente y práctica cotidiana. Revista Iberoamericana de Educación, 55(4), 110.doi:

https://doi.org/10.35362/rie5541580
Bruno, C., Centurión, E., Robleda, G. y Pérez, E. (2015). Diagnóstico de necesidades de capacitación en micro y pequeñas empresas en el municipio de Maxcanú, Yucatán, México. Revista de Administración y Finanzas, 2(2), 304312 . R e c u p e r a d o d e : http://www.ecorfan.org/bolivia/researc hjournals/Administracion_y_Finanzas/ vol2num2/Administracion_Finanzas_V ol2Num2_Final_19.pdf

Curró, C., Pozzolo, O., Fuica, A. y Bogliani, M. (2012). Determinación de las necesidades de capacitación y sus características en la región pampeana y el nordeste de Argentina. X Congreso Latinoamericano y del Caribe de Ingenieria Agrícola - CLIA 2012.15 a 19 de julho - Londrina - PR, Brasil. https://inta.gob.ar/sites/default/files/scri pt-tmp-capacitacion.pdf

FAO. (2018). Diagnóstico de producción ecológica en Bolivia e identificación de necesidades de capacitación. Fundación Agroecol Andes, Ministerio de desarrollo rural y tierras. Cochabamba, Bolivia: FAO. Recuperado de: https://www.agrecolandes.org/wpcontent/uploads/2019/02/diagnosticoproduccion-ecologica_final.pdf

Fernández, J., y Peña R. (2012). Estilos de aprendizaje a partir de la práctica productiva en educación superior rural: caso Utopía. Revista de la Universidad de La Salle, (57), 137-160. Recuperado $\mathrm{d}$ e :

https://ciencia.lasalle.edu.co/cgi/viewc ontent.cgi? article $=1037 \&$ context $=$ ruls

Gajardo, M. (2014). Educación y desarrollo rural en América Latina. Reinstalando un campo olvidado de las políticas educativas. Revista Iberoamericana de Evaluación Educativa, 7(3), 15-27. $\mathrm{R}$ e c u p e r a d o d e : https://revistas.uam.es/index.php/riee/a rticle/view/3099/3298 
García, F. (2012). Campesinado y saber local en la sierra gorda Guanajuatense: procesos de transformación y estrategias de revalorización desde la perspectiva agroecológica (tesis doctoral). Universidad de Córdoba, España.

García, J. (2000). El “estado” de educación rural en América Latina y en África. Revista de Educación, 322 (2000), 119-142. $\mathrm{R}$ e c u p e r a d o d e : http://www.educacionyfp.gob.es/dam/j cr: 9 dfdab 30 - ebc 9 - 46 a e - a 74 f7f6d441c9608/re3220908174-pdf.pdf

Gavito, M., Wal, H., Aldasoro, M., Ayala, B., Bullen, A., Cach, M.,...Villanueva, G. (2017). Ecología, tecnología e innovación para la sustentabilidad: retos y perspectivas en México. Revista mexicana de biodiversidad, 88 (1), 50160 .

https://doi.org/10.1016/j.rmb.2017.09.0 01

Gobierno Mexicano (2007). Sistema nacional de capacitación y asistencia técnica rural integral (SINACATRI). México. Recuperado de:

http://www.inca.gob.mx/pdfinca/Progra ma_Nal_Cap_Rural_Int2007-2012.pdf

Gobierno Peruano (2018). Agenda de conmemoración del bicentenario de la independencia del Perú 2021. Aprobado por D.S. N ${ }^{\circ}$ 009-2018-MC. Recuperado de

https://bicentenariodelperu.pe/wpcontent/uploads/2019/12/DecretoSupremo-009-2018-MC.pdf

Hernández, R., Fernández, C., Baptista, P. (2 014 ). Metodología de la investigación. México: McGraw-Hill.

Hernández, J., Herrera, F. y Chávez, C. (2015). Capacidades, liderazgo y estrategias de gestión de organizaciones de la sociedad civil en zonas rurales. Contaduría y administración 60, 817-835. https://dx.doi.org/10.1016/j.cya.2015.0 7.001

Iglesias, E. (2014). Travesías del desarrollo en Yucatán. Problemas del desarrollo. Revista Latinoamericana de Economía, 45(177), 169-192. Disponible en: https://www.redalyc.org/articulo.oa?id $=118 / 11830741008$

INEI. (2017). Directorio Nacional de Centros Poblados, elaborado en base a Censos Nacionales 2017. XII de población, VII de vivienda y II de comunidades indígenas. Recuperado de: https://www.inei.gob.pe/media/MenuR ecursivo/publicaciones_digitales/Est/Li b1541/index.htm

Iturbide, M.J. (2012). Diagnóstico de Guatemala: necesidades de capacitación para enfrentar los desafios de la negociación internacional de actividades REDD+. Programa REDDCCAD/GIZ. Guatemala. Recuperado d e : http://www.reddccadgiz.org/documento s/doc 423813522.pdf

Juárez, D. (2012). Educación rural en Finlandia: experiencias para México. CPU-e, Revista de Investigación Educativa, 15, $140-154$. R e c u perado d e : http://www.redalyc.org/articulo.oa?id= 283123579008

Landini, F., Bianqui, V. y Russo, M. (2014). Evaluación de un proceso de capacitación para extensionistas rurales implementado en Paraguay. PiracicabaSP, vol 51(1), 09-30. Disponible en: https://www.scielo.br/pdf/resr/v51s1/01 .pdf

Lutz, B. (2017). De la acción colectiva en el campo a la sociedad civil rural. Acta Sociológica núm. 74, 39-76. http://doi.org:10.1016/j.acso.2017.11.0 03 
Martínez, M., Carballo, L. (2013). Educación ambiental rural desde las escuelas básicas y por estas. Revista electrónica educare. 17(2), 69-79. Recuperado de: http://doi.org/10.15359/ree.17-2.4

Maya, C., Ángeles, O. Y Camarena, J. (2014). Diagnóstico de necesidades de educación continua mediante un método grupal y un método individual. Investigación en educación médica, 3(12), 177-186. Recuperado de: http://riem.facmed.unam.mx

Mesa, A. y Machado, H. (2009). Capacitación de productores y directivos para la adopción de tecnologías de producción animal sostenible. Pastos y Forrajes, 32 ( 1$), 1-8$. Re cuperado de: http://scielo.sld.cu/pdf/pyf/v32n1/pyf0 8109.pdf

Monsalvo, A., Jiménez, M., García, J., Sangerman, D., Martínez, T. y Pimentel, J. (2017). Caracterización del perfil del extensionista rural en la zona oriente del Estado de México. Revista Mexicana de Ciencias Agrícolas, 8(3), 503-515. $\mathrm{R}$ e c u p e r a d o d e : http://www.scielo.org.mx/scielo.php?pi $\mathrm{d}=\mathrm{S} \quad 2 \quad 0 \quad 007$ 09342017000300503\&script $=$ sci_artte $\mathrm{xt}$

Mora, J. (2013). Desarrollo rural y ciudadanía social territorios, instituciones y actores locales. San José, Costa Rica: FLACSO. D i s p o n i b l e e n : http://biblioteca.clacso.edu.ar/Costa_Ri c a / fl a c $\begin{array}{llll} & \mathrm{O} & -\end{array}$ cr/20170704034945/pdf_184.pdf

Pastor, G., Abraham, E. y Torres, L. (2005). Desarrollo local en el desierto de Lavalle, estrategia para pequeños productores caprinos (Argentina). Cuadernos de desarrollo rural. (54), $131-149$. Disponible en: https://www.redalyc.org/articulo.oa?id $=117 / 11705407$
Peirano, C., Estévez, S., Astorga, M. (2015). Educación rural: oportunidad para la innovación. Cuadernos de Investigación Educativa, 6(1), 53-70. Recuperado de: http://www.scielo.edu.uy/pdf/cie/v6n1/ v6n1a04.pdf

Posada, M. y Vaca, C. (2001). Diagnóstico sobre necesidades de capacitación en el sector rural. Programa de salud y seguridad en el trabajo del Fundación para la promoción de la seguridad y salud en el trabajo - Fusat. Buenos Aires, Argentina: Fusat. Quispe, A. (2006). La necesidad de formación de capacidades para la gestión del desarrollo rural territorial. Región y sociedad, vol. 18(36). 177-194. Disponible en:

http://www.scielo.org.mx/pdf/regsoc/v 18n36/v18n36a6.pdf

Raczynski, D, Román, M. (2014). Evaluación de la educación rural. Revista Iberoamericana de Evaluación Educativa, 7(3), 9-14. Recuperado de: https://repositorio.uam.es/bitstream/han dle/10486/662838/RIEE_7_3_2.pdf?se quence $=1$

Ramos, R. (2015). Saberes campesinos locales para la interdisciplinariedad educativa rural. Itinerario Educativo, 65, 163-195. Recuperado de: https://revistas.usb.edu.co/index.php/Iti nerario/article/view/1707/1482

Rendón, R., Roldán, E., Hernández, B., y Cadena, P. (2015). Los procesos de extensión rural en México. Revista Mexicana de Ciencias Agrícolas, 6 (1), $151-161$. R e c upe rado de: http://www.scielo.org.mx/scielo.php?sc ript=sci_arttext\&pid=S 2007 $093420150 \overline{0} 0100013$

Rodríguez, C., Sánchez, F., Armenta, A. (2007). Hacia una mejor educación rural: Impacto de un programa de intervención a las escuelas en Colombia. Documente CEDE, 13, 1-34. 